\title{
Rashba precession in quantum wires with interaction
}

\author{
Wolfgang Häusler \\ I. Institut für Theoretische Physik der Universität Hamburg, Jungiusstr. 9, D-20355 Hamburg, Germany
}

(November 13, 2018)

\begin{abstract}
Rashba precession of spins moving along a one-dimensional quantum channel is calculated, accounting for Coulomb interactions. The Tomonaga-Luttinger model is formulated in the presence of spin-orbit scattering and solved by Bosonization. Increasing interaction strength at decreasing carrier density is found to enhance spin precession and the nominal Rashba parameter due to the decreasing spin velocity compared with the Fermi velocity. This result can elucidate the observed pronounced changes of the spin splitting on applied gate voltages which are estimated to influence the interface electric field in heterostructures only little.
\end{abstract}

PACS : 71.10.Pm, 71.70.Ej, 73.20.Dx

According to recent speculations spin could replace the electric charge to carry information in future electronic devices [1]. The 'spin transistor' proposed by Datta and Das [2] might switch faster than traditional transistors since during operation it avoids redistributing charges. The idea is based on the Rashba effect [3] which causes spins to precess as they move along a heterostructure [i] so that the conductance depends on the final spin orientation relative to the magnetization of the ferromagnetic drain contact 5.6 .6. The strength of the Rashba effect is proportional to the electric field acting perpendicular to the electron plane which, when varied by a gate, changes the final spin orientation and thus the transport properties of the device. Advantage is taken of long spin coherence times and lengths [7], found in semiconductors. In the attempt of experimental realization considerable progress has been achieved meanwhile to inject finite spin densities [8]. Polarizations of 90\% have been reported in GaAs [9].

Beating patterns varying with gate voltages have indeed been observed in Shubnikov-de Haas (SdH) measurements 10-12]. Estimates show, however, that the the gate voltage adds an electric field contribution to a much stronger intrinsic field at the interface which does not suffice to explain the observed variations in spin splittings by a factor of two. One should notice that the gate voltage not only changes the strength of the electric field but at the same time it also alters the carrier density in the heterostructure as is directly monitored by the SdH oscillations. Without interactions this would not change the Rashba precession within the effective mass approximation [13] but, since particularly in semiconductors the strength of Coulomb interactions change with density through the $r_{\mathrm{s}}$-parameter, the question arises whether interactions influence the Rashba precession. This is demonstrated in the present Communications. In principle, the two effects of the gate, changing field strength and density, can be separated experimentally by varying the voltage at a front gate and a back gate independently [11].

Usually, Rashba spin precession is described as a band structure effect, resulting from spin splitting [2, [4], as calculated in the original work by Rashba [3] for the homogeneous two-dimensional case. Such a single particle approach, however, cannot account for electron-electron interactions. Including many body effects requires going beyond band structure theory. Particularly convenient 14,15] and powerful 16] is the Tomonaga-Luttinger (TL) model to incorporate interactions exactly for the low energy and long wave length properties in systems of one spatial dimension. From the practical point of view, quantum wires seem most efficient to realize the spin transistor since guiding the electron waves avoids angular dispersion of momenta [2] and diminishes spin scattering events 何. Spin injection into carbon nanotubes, which are known to show pronounced TL behavior [6] has been demonstrated [17]. Here we investigate a quantum wire with spin-orbit interaction in the one-subband limit and apply the TL-model. We expect our main result that interactions enhance Rashba precession to carry over to more channel situations. Indeed, a recent careful analysis within improved versions of the RPA approximation has revealed enhancement of the Rashba precession in the two dimensional electron system [18]. One might comprehend these findings as a consequence of 'repulsion' between the two spin split bands by the interaction.

Spin splitting originates from spin-orbit coupling which in narrow gap heterostructures, such as those based on InAs, is dominated [19] by the Rashba term

$$
H^{\mathrm{so}}=\alpha\left(\sigma_{x} p_{z}-\sigma_{z} p_{x}\right)
$$

where $\sigma_{x, z}$ are Pauli matrices and $p$ is the electron momentum in the $x-z$-plane of the heterostructure. Here, we use coordinates indicated in the inset of Fig. 1. The Rashba parameter $\alpha$ is mostly determined by the intrinsic electric field perpendicular to the interface. Typical values of $1-3 \times 10^{-11} \mathrm{eVm}$ [20] are reported for InAs [10 12].

Taking the confining potential in the lateral $z$-direction as parabolic, the eigenenergies of 


$$
H=\frac{\left(p_{z}+m \alpha \sigma_{x}\right)^{2}+p_{x}^{2}}{2 m}+\frac{m}{2} \omega_{0}^{2} z^{2}-\alpha \sigma_{z} p_{x}
$$

determine the spin split single particle dispersion [21. Here, $\omega_{0}$ is the subband energy and effective mass $(m)$ approximation is assumed. The eigenfunctions are plane waves of momentum $k$ in $x$-direction along the wire and, to good approximation, given as slightly modified oscillator functions $\phi_{n}$ for the subband index $n$

$$
\psi_{k n s}(x, z)=\mathrm{e}^{\mathrm{i} k x} \phi_{n}(z)(\cos (m \alpha z)|s\rangle+\mathrm{i} \sin (m \alpha z)|-s\rangle) .
$$

On the wire axis the $\psi_{k n s}(x, z)$ are spin polarized along the $z$-direction $(s= \pm 1)$ while the spins acquire a non-zero out of plane $y$-component $\sim \sin (2 m \alpha z)=\sin (2 \sqrt{\eta} z / \ell)$ away from the axis. This describes a texture in the lateral spin density distribution. Here, $\ell=\left(m \omega_{0}\right)^{-1 / 2}$ is the oscillator length and the parameter $\eta=\frac{m}{\omega_{0}} \alpha^{2}=\frac{2 \varepsilon_{\mathrm{F}}}{\omega_{0}}\left(\alpha / v_{\mathrm{F}}\right)^{2}$ is introduced for later use $\left(\varepsilon_{\mathrm{F}}\right.$ is the Fermi energy above the subband edge and $v_{\mathrm{F}}$ the Fermi velocity). In the mostly used compounds $\alpha / v_{\mathrm{F}}<0.2$ [11] (in GaAs it is even much smaller), so that always $\eta<0.1$ in the lowest subband.

At $k=0$ different spin orientations are degenerate but when $k \neq 0$ Rashba splitting occurs, yielding

$$
E_{k 0 s}=-m^{*} \alpha^{* 2}+\frac{\omega_{0}}{2}+\frac{1}{2 m^{*}}\left(k+s m^{*} \alpha^{*}\right)^{2}, \quad s= \pm 1
$$

for the energies of the lowest subband. Expression (何) is obtained using the basis (3) after expanding Laguerre polynomials arising from band mixing by the spin-orbit term in powers of $\eta$. Up to the order $\mathcal{O}\left(\eta^{5 / 2}\right)$ one can incorporate the effect of subband mixing, described by $\eta$, into renormalized values for effective mass $m^{*}=m\left(1+8 \eta^{2}\right)$ and Rashba parameter $\alpha^{*}=\alpha(1-\eta)$. Note that a similar reasoning would be true also for other than parabolic dispersion relations where effects of inter-subband mixing could still be incorporated into renormalized values for the kinetic energy parameter and for $\alpha$. The dispersion relation (4) resembles the one obtained in two dimensions [3] but restricted to one dimensional $k$-space, cf. Fig. 1. For higher subbands the leading energy correction $\sim 2 n \eta$, even in the strictly parabolic dispersion case, depends on the subband index $n$ which somewhat weakens the optimistic conjecture expressed by Datta and Das [2] that the proposed spin transistor would not loose sensitivity in multi-mode operation or at elevated temperatures or voltages. The Rashba phases in higher subbands differ slightly, $\sim \eta$, from the phase acquired in the ground subband.

Without interactions the difference $2 m^{*} \alpha^{*}$ in momenta between $s=+1$ and $s=-1$ carriers, being independent of Fermi energy, is the origin for the Rashba precession of spins initially polarized e.g. along the $+x$-direction. After traversing the Rashba distance $\lambda_{\mathrm{R}}=\pi / m^{*} \alpha^{*}$ the spin is reversed. Contrary to the observations [10 12], Rashba splitting, and therefore $\lambda_{\mathrm{R}}$, is independent of carrier density $2 k_{\mathrm{F}} / \pi$ in (4) at given interfacial electric field [13]; $k_{\mathrm{F}}$ is the Fermi momentum. Also, we note that the Fermi velocities of both spin components are equal.

The term $\sim s m^{*} \alpha^{*}$ inside the brackets in Eq. (4), shifting particle momentum, formally resembles a vector potential associated with the magnetic flux through an Aharanov-Bohm ring, leading there [22 23] to a persistent charge current in the ground state. Similarly, in (4i) this term causes a non-zero persistent spin current $\sim\left\langle J_{\sigma}\right\rangle$ when periodic boundary conditions are imposed 24].

This similarity carries over to the TL-model for the quantum wire at low energies which in Bose variables reads

$$
H=\sum_{\nu=\rho, \sigma} \frac{\pi}{4 L}\left(v_{\nu \mathrm{N}} N_{\nu}^{2}+v_{\nu \mathrm{J}} J_{\nu}^{2}\right)+\left(\sum_{q \neq 0} H_{q}\right)-m^{*} \alpha^{*} v_{\mathrm{F}} J_{\sigma}
$$

Here, $N_{\nu}=N_{\nu \mathrm{R}}+N_{\nu \mathrm{L}}$ and $J_{\nu}=N_{\nu \mathrm{R}}-N_{\nu \mathrm{L}}$ denote particle numbers and currents, respectively, $N_{\mathrm{L} / \mathrm{R}}$ are the number of left/right going particles; $L$ is the wire length. Topological excitations of $N_{\nu}$ or $J_{\nu}$ as well as density excitations at momentum $q$, described by $H_{q}$, preserve charge $(\nu=\rho)$ spin $(\nu=\sigma)$ separation (as already mentioned, here we have to high accuracy equal velocities of both spins, cf. [21]; this is not the case, for example, in the presence of a Zeeman field [25], when spin up and spin down velocities differ at the Fermi energy due to the quadratic energy dispersion). The interaction is exactly included in the TL-model by renormalized values for its parameters such as the velocities $v_{\nu \mathrm{N}}$ and $v_{\nu \mathrm{J}}$ which now differ from $v_{\mathrm{F}}$. In (5) we have already omitted the backscattering in spin sector originating from the exchange of two electrons near two opposite Fermi points of opposite spins. Such a term would couple the topological sector with the charge and spin density excitations. For repulsive interactions back scattering is known to be irrelevant at low energies [26], also when persistent currents are present [23]. Therefore, the $H_{q}$ will decouple, leaving the the correlation exponents $K_{\nu}\left(K_{\nu} \rightarrow 1\right.$ without interactions) and the power law decays of various correlation functions, that can be calculated within the TL-model, unaffected [27] by the Rashba coupling $\sim \alpha$, cf. Eq. (6) below, contrary to the result obtained in Ref. [21]. Spin-orbit coupling, and therefore the Rashba term (the last term in (5)), does not depend explicitly on the interaction. 
Electrons injected at $x=0$ with spins polarized along the wire axis will perform Rashba precession. Most directly this can be monitored using the correlation function $f(x)=\frac{1}{2}\left\langle\left(\Psi_{\uparrow}(x)+\Psi_{\downarrow}(x)\right)\left(\Psi_{\uparrow}^{+}(0)+\Psi_{\downarrow}^{+}(0)\right)\right\rangle$ where the Fermi operator $\Psi_{s}(x) \propto \mathrm{e}^{-\mathrm{i} \sqrt{\pi / 2}\left(\phi_{\rho}(x)+s \phi_{\sigma}(x)\right)} \sum_{r= \pm} \mathrm{e}^{-\mathrm{i} r \sqrt{\pi / 2}\left(\theta_{\rho}(x)+s \theta_{\sigma}(x)\right)}$ is expressed through the momentum

$$
\phi_{\nu}(x)=\sqrt{\frac{\pi}{2}} J_{\nu} \frac{x}{L}+\sum_{q \neq 0} \phi_{\nu q}(x)
$$

and the density like Bose fields

$$
\theta_{\nu}(x)=\sqrt{\frac{\pi}{2}} N_{\nu} \frac{x}{L}+\sum_{q \neq 0} \theta_{\nu q}(x)
$$

in the usual way 14, 15. The $q \neq 0$ components yield power law decay while the $q=0$ components are relevant for charge or spin stiffness and the persistent current. The result for the desired correlation function is

$$
\begin{aligned}
f(x)= & -\frac{k_{\mathrm{F}}}{\pi}\left|k_{\mathrm{F}} x\right|^{-\left(K_{\rho}+1 / K_{\rho}+K_{\sigma}+1 / K_{\sigma}\right) / 4} \\
& \times \sin \left(\left|k_{\mathrm{F}} x\right|\right) \cos \left(\frac{\pi}{2} j_{\sigma} x\right) .
\end{aligned}
$$

The square $|f(L)|^{2}$ is proportional to the probability for spin polarization in $+x$-direction at a distance $L$ from the source and, by a similar reasoning as in [2], to the current accepted by an ideal ferromagnetic drain which is polarized parallel to the source in $+x$-direction. A more sophisticated full transport calculation has been carried out recently [6] in the absence of spin-orbit coupling but would go beyond the scope of the present work. The period in the slowly oscillating last term in (6), $j_{\sigma} \equiv\left\langle J_{\sigma}\right\rangle / L=\frac{2}{\pi} m^{*} \alpha^{*} v_{\mathrm{F}} / v_{\sigma \mathrm{J}}$, where the expectation value $\langle$.$\rangle refers to the ground state$ of (5), determines the Rashba length

$$
\lambda_{\mathrm{R}}=\frac{\pi}{m^{*} \alpha^{*}} \frac{v_{\sigma \mathrm{J}}}{v_{\mathrm{F}}} .
$$

This quantity depends on the velocity of the spin current $v_{\sigma \mathrm{J}}$ and is now altered compared to its value in the absence of interactions. We note, that in charge sector the corresponding velocity $v_{\rho \mathrm{J}}=v_{\mathrm{F}}$ remains constant as a consequence of Galilei invariance of the quantum wire [15] at carrier densities much smaller than the inverse lattice constant of the underlying semiconductor lattice. Galilei invariance does not hold in spin sector where particles of opposite spins moving in opposite directions will experience some drag by the interaction. Therefore, $v_{\sigma \mathrm{J}}$ differs from $v_{\mathrm{F}}$.

Rigorous relations hold [14] among the velocities $v_{\nu \mathrm{s}}=v_{\nu} K_{\nu}=v_{\nu \mathrm{N}} K_{\nu}^{2}$ in TL-liquids, determined by the correlation exponents. Here, $v_{\nu}$ is the velocity of density excitations at small $q$. In many cases spin rotation invariance holds and fixes $K_{\sigma}=1$. Rashba coupling breaks this $\mathrm{SU}(2)$ invariance. Fortunately, the corresponding energy $2 m^{*} \alpha^{*} v_{\mathrm{F}}$ is small compared to the Fermi energy (or to the typical Coulomb energy $v_{\rho \mathrm{N}} / v_{\mathrm{F}}$ ) so that $K_{\sigma}$ will deviate from unity at most slightly and $v_{\sigma \mathrm{J}} \approx v_{\sigma} \approx v_{\sigma \mathrm{N}}$ to good accuracy. Much more important than the differences between those three velocities are their deviation from $v_{\mathrm{F}}$ occurring already in the $\mathrm{SU}(2)$ symmetric case as seen for example in the Hubbard chain [28]. How long range interactions alter $v_{\sigma} / v_{\mathrm{F}}$ has been investigated perturbatively [29] and, recently, by extensive quantum Monte Carlo studies [30]. Starting from values close to unity at high carrier densities, $r_{\mathrm{s}}<0.5$, $v_{\sigma} / v_{\mathrm{F}}$ can drop below 0.5 when $r_{\mathrm{s}} \gtrsim 1.4$. Those densities are easily reached in present day quantum wires $[16]$. In the limit of very small particle densities $2 k_{\mathrm{F}} / \pi$ the spin velocity is expected to vanish like $v_{\sigma} \sim k_{\mathrm{F}}^{2}$.

Thus, according to (7), Rashba precession is enhanced by repulsive interactions. Qualitatively, one might understand this result on the mean field level as a consequence of the Fock contribution precipitating repulsions between opposite rather than same spins. This enhances spin splitting and the nominal value of the Rashba parameter. In principle this argument applies also to situations with more than one subband occupied or even two dimensional heterostructures. Also in those cases we would expect that Coulomb interactions amplify Rashba precession as it has been confirmed in Ref. [18].

The amount of Rashba enhancement expected at given carrier density can be measured independently through the Zeeman spin susceptibility $\chi=-\left(\partial^{2} E_{0} / \partial B^{2}\right) / L=\left(L \partial^{2} E_{0} / \partial N_{\sigma}^{2}\right)^{-1}=2 / \pi v_{\sigma N}$ by monitoring the 'exchange enhancement' of the effective $g$-factor; $E_{0}$ is the ground state energy of (5). In InAs the magnetic energy $B$ translates quite accurately as 1 Ry per Tesla when taking the $g$-factor [31] as $|g|=13$. Assuming again $K_{\sigma} \approx 1$ yields

$$
\lambda_{\mathrm{R}}=2 / m^{*} \alpha^{*} v_{\mathrm{F}} \chi .
$$


In conclusion, we have established a theory beyond describing Rashba precession as a single particle band structure effect. We have considered a quantum wire and the TL-model to incorporate interactions exactly. Increasing repulsion between carriers along with decreasing particle densities is found to reduce the Rashba length $\lambda_{\mathrm{R}}$ over which spins complete cycles as they move along the wire. Accordingly, the nominal value of the Rashba parameter increases, as determined by Shubnikov-de Haas measurements. This is demonstrated to be a consequence of decreasing spin velocities. The latter could be measured independently through the magnetic susceptibility w.r.t. a Zeeman field. Contrary to the relatively small influence of gates on the strength of the interfacial electric field this interaction induced contribution can explain variations of $\lambda_{R}$ by a factor of 2 . It would be valuable to experimentally separate the influence of the field strength from the carrier density by applying voltages independently to a front and a back gate [11]. Moreover, the front gate could screen the long range part of the Coulomb interaction and thereby serve to vary the microscopic interaction strength.

I thank Charles Creffield, Dirk Grundler, Allan MacDonald, Ulrich Merkt, and, especially, Ulrich Zülicke for valuable discussions, and M. E. Raikh for drawing my attention to the work of Ref. [18.

[1] G.A. Prinz, Physics Today, Apr. 1995, p. 58.

[2] B. Datta and S. Das, Appl. Phys. Lett. 56, 665 (1990).

[3] É.I. Rashba, Fiz. Tverd. Tela 2, 1224 (1960) [Sov. Phys. Solid State 2, 1109 (1960)]; Yu.A. Bychkov and É.I. Rashba, Pis'ma Zh. Eksp. Teor. Fiz. 39, 66 (1984) [JETP Lett. 39, 78 (1984)].

[4] A. Bournel, P. Dollfus, P. Bruno, and P. Hesto, Euro. Phys. J. Appl. Phys. 4, 1 (1998).

[5] A. Brataas, Yu.V. Nazarov, and G.E.W. Bauer, Phys. Rev. Lett. 84, 2481 (2000).

[6] L. Balents and R. Egger, Phys. Rev. Lett. 85, 3464 (2000).

[7] D. Hägele, M. Oestreich, W.W. Rühle, N. Nestle, and K. Eberl, Appl. Phys. Lett. 73, 1580 (1998); J.M. Kikkawa and D.D. Awschalom, Nature 397, 139 (1999).

[8] Y. Ohno, D.K. Young, B. Beschoten, F. Matsukura, H. Ohno, and D.D. Awschalom, Nature 402, 790 (1999); M. Oestreich, J. Hübner, D. Hägele, P.J. Klar, W. Heimbrodt, W.W. Rühle, D.E. Ashenford, and B. Lunn, Appl. Phys. Lett. 74, 1251 (1999).

[9] R. Fiederling, M. Keim, G. Reuscher, W. Ossau, G. Schmidt, A. Waag, and L.W. Molenkamp, Nature 402, 787 (1999).

[10] J. Nitta, T. Akazaki, H. Takayanagi, and T. Enoki, Phys. Rev. Lett. 78, 1335 (1997); G. Engels, J. Lange, T. Schäpers, and H. Lüth, Phys. Rev. B 55, R1958 (1997); T. Schäpers, G. Engels, J. Lange, T. Klocke, M. Hollfelder, and H. Lüth, J. Appl. Phys. 83, 4324 (1998); C.-M. Hu, J. Nitta, T. Akazaki, H. Takayanagi, J. Osaka, P. Pfeffer, and W. Zawadzki, Phys. Rev. B 60, 7736 (1999).

[11] D. Grundler, Phys. Rev. Lett. 84, 6074 (2000).

[12] T. Matsuyama, R. Kürsten, C. Meißner, and U. Merkt, Phys. Rev. B 61, 15588 (2000).

[13] In narrow gap semiconductors with almost linear single particle dispersion the Rashba precession would decrease with decreasing electron density which is opposite to what is predicted here as a consequence of interaction effects.

[14] F.D.M. Haldane, J. Phys. C 14, 2585 (1981).

[15] J. Voit, Rep. Prog. Phys. 58, 977 (1995).

[16] A. Yacoby, H.L. Stormer, N.S. Wingreen, L.N. Pfeiffer, K.W. Baldwin, and K.W. West, Phys. Rev. Lett. 77, 4612 (1996); M. Rother, W. Wegscheider, R.A. Deutschmann, M. Bichler, and G. Abstreiter, Physica E (Amsterdam) 6, 551 (2000).

[17] K. Tsukagoshi, B.W. Alphenaar, and H. Ago, Nature (London) 401, 572 (1999).

[18] G.-H. Chen and M. E. Raikh, Phys. Rev. B 60, 4826 (1999).

[19] G. Lommer, F. Malcher, and U. Rössler, Phys. Rev. Lett. 60, 728 (1988); U. Rössler, F. Malcher, and G. Lommer in "High magnetic fields in Semiconductor Physics II", Vol. 87, Springer Series in Solid State Sciences, ed. by G. Landwehr (Springer, Berlin, 1989).

[20] In the present work we consider $\alpha$ as being defined through (1) while $2 \lambda_{\mathrm{R}}$ is the observed spatial length carriers have to traverse to perform one cycle in spin. Thus $\lambda_{\mathrm{R}}$ may depend on particle density while $\alpha$ does not.

[21] The work by A.V. Moroz, K.V. Samokhin, and C.H.W. Barnes, Phys. Rev. Lett. 84, 4164 (2000) does not resolve the here posed question how increasing interaction strength influences the Rashba precession.

[22] D. Schmeltzer, Phys. Rev. B 47, 7591 (1993).

[23] D. Loss, Phys. Rev. Lett. 69, 343 (1992).

[24] Y. Meir, Y. Gefen, and O. Entin-Wohlman, Phys. Rev. Lett. 63, 798 (1989).

[25] T. Kimura, K. Kuroki, and H. Aoki, Phys. Rev. B 53, 9572 (1996).

[26] J. Sólyom, Adv. in Phys. 28, 201 (1979).

[27] Actually, there is a non-zero change of the TL-parameters also in the charge sector, describing density excitations, due to 
the Rashba term since the Coulomb matrix elements are slightly changing with the wave functions (3). This effect, however, is much smaller than the influence of a changing carrier density and can safely be ignored.

[28] H.J. Schulz, Phys. Rev. Lett. 64, 2831 (1990).

[29] H.J. Schulz, Phys. Rev. Lett. 71, 1864 (1993).

[30] C.E. Creffield, W. Häusler, and A.H. MacDonald, Europhys. Lett. 53, 221 (2001); W. Häusler, L. Kecke, and A.H. MacDonald (unpublished).

[31] S. Brosig, K. Ensslin, A.G. Jansen, C. Nguyen, B. Brar, M. Thomas, and H. Kroemer, Phys. Rev. B 61, 13045 (2000). 


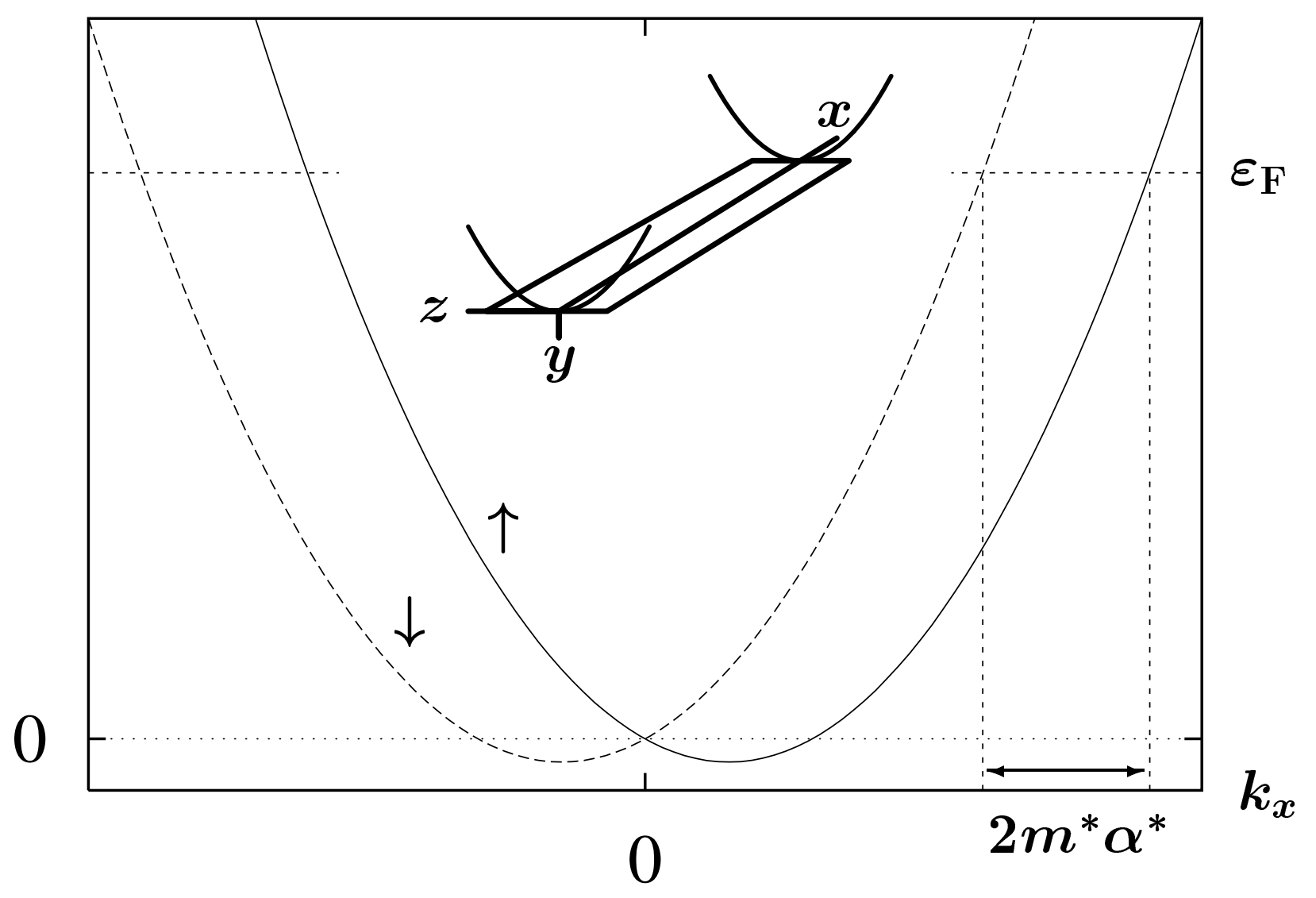

FIGURE 1

Fig. 1: Energy dispersion in the lowest spin split subband of a quantum wire with parabolic confining potential. On the wire axis the spins $s=\uparrow, \downarrow$ are polarized in the plane of the heterostructure. 